\title{
Markers and risk factors for chronic kidney disease in sub-Saharan Africans: baseline levels and 12-month trajectories in newly referred patients in Cameroon
}

Halle Marie Patrice ${ }^{1 *}$, Kengne Andre Pascal ${ }^{2}$, Kaze Folefack François ${ }^{3}$, Djantio Hilaire ${ }^{4}$, Doualla Marie Solange ${ }^{5}$, Ashuntantang Enow Gloria ${ }^{6}$ and Choukem Siméon Pierre ${ }^{7}$

\begin{abstract}
Background: Little is known about the changes in disease makers and risk factors in patients with chronic kidney disease (CKD) under nephrological care in Africa. This study aimed to evaluate the baseline level of markers of CKD and their 12-month time-trend in newly referred patients in a tertiary hospital in Cameroon.

Methods: This was a retrospective cohort study including 420 patients referred for CKD between 2006 and 2012 to the nephrology unit of the Douala General Hospital in the littoral region of Cameroon. Their disease and risk profile was assessed at baseline and every 3 months for 1 year. Estimated glomerular filtration rate (eGFR) was based on MDRD and Schwartz equations. CKD was diagnosed in the presence of eGFR $<60 \mathrm{ml} / \mathrm{min} / 1.73 \mathrm{~m}^{2}$ and/or proteinuria $>1+$ and/or abnormal renal ultrasound persisting for $\geq 3$ months. Data analysis used mixed linear regressions.
\end{abstract}

Results: Of the 420 patients included, 66.9\% were men and mean age was 53.8 (15.1) years. At referral, 37.5\% of the participants were at CKD Stage 3,30.8\% at stage 4 and 26.8\% at stage 5. There was 168 (40\%) diabetic and 319 (75.9\%) hypertensive patients. After some improvement during the first 3 months, eGFR steadily decreased during the first year of follow-up, and this pattern was robust to adjustment for many confounders. Systolic and diastolic blood pressure levels significantly fluctuated during the first twelve months of follow-up. Changes in the levels of other risk factors and markers of disease severity over time were either borderline or non-significant.

Conclusion: Patients with CKD in African settings are referred to the nephrologist at advanced stages. This likely translates into a less beneficial effects of specialised care on the course of the disease.

Keywords: Risk factor, Chronic kidney disease, Trajectory, Cameroon

\section{Background}

Chronic kidney disease (CKD) is a public health problem worldwide, with growing prevalence estimated at 11 to

\footnotetext{
* Correspondence: patricehalle@yahoo.fr

'Department of internal medicine Douala General Hospital Cameroon Faculty of medicine and pharmaceutical science, University of Douala, Douala, Cameroon

Full list of author information is available at the end of the article
}

$13 \%$ in the adult population [1]. It has sustainably been a major contributor to the global burden of disease in the last two decades [2]. Main aetiologies of CKD worldwide are diabetes mellitus, hypertension and chronic glomerulonephritis [3]. CKD is characterized by 5 stages of irreversible impaired renal function, with progressive decline towards end stage kidney disease (ESKD) requiring renal replacement therapy (RRT). The rate of

C C The Author(s). 2020 Open Access This article is licensed under a Creative Commons Attribution 4.0 International License, which permits use, sharing, adaptation, distribution and reproduction in any medium or format, as long as you give appropriate credit to the original author(s) and the source, provide a link to the Creative Commons licence, and indicate if changes were made. The images or other third party material in this article are included in the article's Creative Commons licence, unless indicated otherwise in a credit line to the material. If material is not included in the article's Creative Commons licence and your intended use is not permitted by statutory regulation or exceeds the permitted use, you will need to obtain permission directly from the copyright holder. To view a copy of this licence, visit http://creativecommons.org/licenses/by/4.0/. The Creative Commons Public Domain Dedication waiver (http://creativecommons.org/publicdomain/zero/1.0/) applies to the data made available in this article, unless otherwise stated in a credit line to the data. 
progression depends on comorbidities and risk factors. Effective strategies can slow the progression of CKD and may help reducing the risk of cardiovascular disease (CVD) and death $[4,5]$.

CKD disproportionately affects African descendants [6-8]. This is due to increased prevalence among Africans, of known risk factors for CKD such as diabetes, hypertension, genetic polymorphisms such as Apolipoprotein L1, and sickle cell trait [9-12]. CKD progresses more rapidly in people of African ethnicity [13-16]. In sub-Saharan Africa (SSA), CKD affects $12-23 \%$ adults [17-19], and mostly in their young and productive age [12, 20-23]. Despite the benefit of early referral on CKD progression, the rate of late referral of patients to the nephrologist is extremely high in SSA $[24,25]$, where access to RRT is limited [26]. Patients with CKD therefore face the problems of high out-of-pocket payment and poor outcome on RRT [27-29].

Few studies have reported the baseline profile of patients with CKD at referral and in SSA [23, 24, 28, 29]. Little is known on the evolution of their kidney function, related risk factors and markers of CKD progression under nephrological care. The objective of this study was to evaluate the baseline level of markers of CKD and their 12-month time-trend in newly referred patients at a tertiary hospital in Cameroon.

\section{Methods}

\section{Study setting}

This retrospective cohort study was based on registry and files of the out-patient section of the nephrology unit of the Douala General Hospital (DGH) in Cameroon. DGH is a 320-bedded public institution, serving as referral hospital for kidney disease for the Littoral region of the country and beyond. It has the largest haemodialysis unit of the country, and provides ongoing RRT to about 230 patients. The medical staff of the unit comprises two nephrologists, one general practitioner and twelve nurses. Patients with CKD referred to the unit are assigned a unique identifier and attached to one of the nephrologists, and then followed-up at intervals that are determined by the stage of the renal disease. At the first consultation in the unit, each patient has clinical assessment and laboratory tests done. The diagnosis of kidney disease was based on estimated glomerular filtration rate less than $60 \mathrm{ml} / \mathrm{min}$ and /or proteinuria. The aetiology of CKD was mostly based on clinical arguments. Patients are generally referred at the advanced stage of CKD when shrunken kidneys preclude any reliable histological diagnosis. Among those eventually eligible for such diagnosis, renal biopsy is seldom done in the unit. Ethical approval was obtained from the ethical committee board of the Douala University and administrative authorization from the DGH.

\section{Study participants}

In the present study, we included all patients referred for CKD between January 2006 and December 2012. We did not include patients on renal replacement therapy in this study. Socio-demographic characteristics such as age and sex, and relevant clinical data including existing hypertension, diabetes mellitus, HIV, gout, and medication at referral were recorded. Blood pressure, aetiology of CKD, biological parameters including serum urea and creatinine level, glycaemia, uric acid, lipid profile, serum albumin and haemoglobin level were noted for the baseline level and every 3 months during the first 12 months of follow-up.

\section{Definitions}

The abbreviated version of the Modification of Diet in Renal Disease (MDRD) and Schwartz equations were used for estimated glomerular filtration rate (eGFR) in patients aged $\geq 18$ Years and $<18$ years respectively [ 30 , 31]. CKD was defined by eGFR $<60 \mathrm{ml} / \mathrm{min} / 1.73 \mathrm{~m}^{2}$ and $/$ or proteinuria $>1+$ and/or abnormal renal ultrasound (small shrunken, polycystic or asymmetric kidney), persisting for $\geq 3$ months. Patients were classified following the Kidney Disease Improving Global Outcome (KDIGO) staging of CKD [32]. CKD stage 1: eGFR $\geqq 90$ $\mathrm{mL} / \mathrm{min} / 1.73 \mathrm{~m}^{2}$ with proteinuria or abnormal kidney, stage 2: $60 \leqq \mathrm{eGFR}<90 \mathrm{~mL} / \mathrm{min} / 1.73 \mathrm{~m}^{2}$ with proteinuria and /or abnormal kidney, stage 3: $30 \leqq \mathrm{eGFR}<60 \mathrm{~mL} /$ $\min / 1.73 \mathrm{~m}^{2}$, stage $4: \quad 15 \leqq \mathrm{eGFR}<30 \mathrm{~mL} / \mathrm{min} / 1.73 \mathrm{~m} 2$, stage 5: eGFR $<15 \mathrm{~mL} / \mathrm{min} / 1.73 \mathrm{~m}^{2}$. Diabetes was defined by a fasting serum glucose $\geqq 126 \mathrm{mg} / \mathrm{dL}$, or random glucose $\geqq 200 \mathrm{mg} / \mathrm{dL}, \quad \mathrm{HbA} 1 \mathrm{c} \geqq 6.5 \%$, or the use of hypoglycaemic agents. Hypertension at referral was defined as systolic blood pressure (SBP) $\geq 140 \mathrm{mmHg}$, diastolic blood pressure (DBP) $\geq 90 \mathrm{mmHg}$, or use of antihypertensive agents.

\section{Statistical analysis}

Data analysis used SAS STAT v 9.1 for Windows ${ }^{\circ}$ (SAS Institute Inc., Cary, NC, USA). We have reported baseline characteristics as count and percentages, and mean and standard deviation, and compared them across major subgroups via chi square tests and equivalents for qualitative variables, and Student's t-test for continuous variables. Mixed linear regression models were used to examine changes in kidney function, determinants and indicators of disease complications (severity) during the first 12 months of follow-up while adjusting for baseline and changing levels of potential confounders during follow-up. Heterogeneity in the trajectories of key outcomes across major subgroups was investigated through interaction tests. Mixed linear models are suitable for handling longitudinal data with repeated measurements on continuous outcomes, particularly when there are 
missing data, which is rather a common situation in observational studies like this one. A $p$-value $<0.05$ was used to indicate statistically significant results.

\section{Results}

Baseline characteristics of participants overall and by sex A total of 420 patients were included; of whom $66.9 \%$ were men. The mean age (standard deviation) was 53.8 (15.1) years, with no significant difference in age between men and women $(p=0.09)$. The mean eGFR at referral was $28.6(17.0) \mathrm{ml} / \mathrm{min} / 1.73 \mathrm{~m}^{2}$ overall, 30.9 (17.8) in men and $24.0(14.2) \mathrm{ml} / \mathrm{min} / 1.73 \mathrm{~m}^{2}$ in women $(p<$ 0.0001). The staging of kidney function at referral was: Stage 1 in $0.7 \%$ of participants, Stage 2 in $4.2 \%$, Stage 3 in $37.5 \%$, Stage 4 in $30.8 \%$ and Stage 5 in $26.8 \%$; with a borderline significant difference by gender $(p=0.04)$. There was 168 (40\%) diabetic, 319 (75.9\%) hypertensive and 20 (4.9\%) HIV positive patients at referral. Average blood pressure (BP) and creatinine levels were high, mostly similarly in men and women (all $p>0.39$ ), while haemoglobin level was low, and much so in women $(p=$ 0.0006) and the distribution of other hematologic parameters, electrolytes, lipid profile showed no major gender differences. Men were more likely to be smokers $(p=0.025)$, alcohol drinker $(p<0.0001)$, physically active $(p=0.001)$, to have gout $(p=0.011)$ and less likely to be HIV positive $(p=0.009)$ compared with women. Treatments, including for co-morbidities did not differ between men and women (Table 1).

\section{Baseline characteristics in other major subgroups of participants}

Baseline differences were apparent between participants with diabetes and those without, with regard to age $(p<$ $0.0001)$, diastolic blood pressure $(p=0.0002)$, potassium $(p=0.002)$, uric acid $(p=0.028)$, prevalent hypertension $(p<0.0001)$, stage of kidney function $(p=0.013)$, treatment with ACE inhibitors $(p=0.011)$ or ARA II $(p=$ 0.043). Compared with participants without hypertension, those with hypertension were more likely to be older, to have higher weight, creatinine, haemoglobin, albumin and phosphate levels (all $p \leq 0.043$ ). They were also more likely to have diabetes $(p<0.0001)$, to be alcohol drinkers $(p=0.033)$, sedentary $(p=0.005)$, to comprise fewer people with HIV infection $(p<0.0001)$, to be referred with advanced stage CKD $(p=0.002)$.

\section{Trajectories of kidney function during follow-up}

After some improvement during the first 3 months of follow-up, eGFR steadily decreased during the first twelve months of follow-up, and this pattern was robust to adjustment for age, sex, status for hypertension, diabetes mellitus, smoking, alcohol consumption and HIV infection $(p=0.003)$; Table 2 and Fig. 1. In similar analyses stratified by baseline status for diabetes (Table 3) or hypertension (Table 4), the pattern was mostly similar with however a significant effect only in participants with diabetes $(p=0.013)$ but not in those without $(p=$ 0.205). While there was no evidence of statistical interaction by diabetes status in the trajectories of eGFR (interaction $p=0.646$ ), a borderline interaction was apparent in the effects by status for hypertension $(p=$ $0.054)$, primarily driven by an improvement in the eGFR between 9 and 12 months of follow-up among participants without hypertension at baseline (Fig. 1). Serum urea level did not change significantly during follow-up overall and within major subgroups of participants.

\section{Trajectories of other markers and risk factors for CKD}

Systolic and diastolic BP levels significantly fluctuated during the first twelve months of follow-up, with both ups and downs observed between consecutive visits (both $p<$ 0.0001); (Table 2), with suggestions that these fluctuations occurred in a differential way for SBP between participants with diabetes and those without (interaction $p=0.006$ ); (Table 3$)$, but not for DBP $(p=0.174)$, nor by status for hypertension (both interaction $p \geq 0.355$ ); (Table 4). Haemoglobin levels decreased between baseline and 3month visit, and steadily increased thereafter, although the overall effect was not significant $(p=0.09)$. This pattern was consistent by status hypertension, while differing trajectories were observed among participants with diabetes (significant increase over time, $p=0.022$ ) and those without diabetes (borderline significant decreased over time, $p=0.079$ ), with significant statistical interaction (interaction $p=0.005$ ). Other haematological parameters, electrolytes and lipid profile did not change significantly during follow-up overall and within major subgroups of participants.((Tables 2, 3 and 4)

\section{Discussion}

In this study, we have for the first time described the time-trend in the trajectory of kidney function, risk makers and health consequences in patients with CKD in a SSA setting. We found a deteriorating kidney function over time, which was robust to adjustment for potential confounders and broadly similar across levels of major risk factors such as diabetes mellitus and hypertension; although some late improvement was observed among non-hypertensive participants, resulting in borderline interaction by status for hypertension. We found fluctuating levels of blood pressure over time, which was significantly different by status for diabetes, but no for hypertension, and likely reflecting the difficulties to achieve and maintain adequate blood pressure control in patients with CKD. Changes in the levels of other risk factors and markers of disease severity over time were either borderline or non-significant. 
Table 1 Baseline characteristics overall and by major subgroups

\begin{tabular}{|c|c|c|c|c|c|c|c|c|c|c|}
\hline Characteristics & Overall & Men & Women & $p$ & HTN & No HTN & $P$ & DM & No DM & $p$ \\
\hline$\overline{N(\%)}$ & $420(100)$ & $281(66.9)$ & $139(33.1)$ & & $319(76.0)$ & $101(24.0)$ & & $168(40.0)$ & $252(60.0)$ & \\
\hline Gender, men (\%) & $281(66.9)$ & $281(100)$ & $0(0)$ & & $221(69.3)$ & $60(59.4)$ & 0.070 & $118(70.2)$ & $163(64.7)$ & 0.246 \\
\hline Age, years (SD) & $53.8(15.1)$ & $54.7(14.6)$ & $52.1(15.8)$ & 0.097 & $57.9(11.2)$ & $41.1(18.2)$ & $\begin{array}{l}< \\
0.0001\end{array}$ & $60.7(8.2)$ & $49.3(16.6)$ & $\begin{array}{l}< \\
0.0001\end{array}$ \\
\hline Weight, kg (SD) & $76.5(16.3)$ & $78.4(17.7)$ & $72.7(12.5)$ & 0.003 & $79.6(14.5)$ & $68.3(18.1)$ & $\begin{array}{l}< \\
0.0001\end{array}$ & $78.1(15.2)$ & $75.4(17.0)$ & 0.206 \\
\hline $\mathrm{SBP}, \mathrm{mmHg}(\mathrm{SD})$ & $159(31)$ & $160(30)$ & $157(34)$ & 0.392 & $164(30)$ & $140(27)$ & $\begin{array}{l}< \\
0.0001\end{array}$ & $162(29)$ & $157(32)$ & 0.087 \\
\hline $\mathrm{DBP}, \mathrm{mmHg}(\mathrm{SD})$ & $93(18)$ & $93(18)$ & $92(18)$ & 0.668 & $94(19)$ & $87(16)$ & 0.0004 & $89(16)$ & $96(19)$ & 0.0002 \\
\hline Urea & $0.88(0.56)$ & $0.84(0.52)$ & $0.96(0.62)$ & 0.064 & $0.90(0.53)$ & $0.79(0.61)$ & 0.084 & $0.92(0.50)$ & $0.85(0.60)$ & 0.192 \\
\hline Creatinin & $36.9(27.4)$ & 36.7 (27.6) & $37.3(27.2)$ & 0.831 & $39.2(28.7)$ & $29.5(21.2)$ & 0.0004 & $35.5(23.8)$ & 37.9 (29.6) & 0.367 \\
\hline eGFR & $28.6(17.0)$ & $30.9(17.8)$ & $24.0(14.2)$ & $\begin{array}{l}< \\
0.0001\end{array}$ & $26.9(15.1)$ & $34.9(21.4)$ & 0.001 & $27.8(13.9)$ & $29.2(18.9)$ & 0.387 \\
\hline Sodium & $138.7(10.4)$ & $138.6(10.6)$ & $139.1(9.8)$ & 0.691 & $139.1(11.4)$ & $137.6(5.7)$ & 0.136 & $137.9(14.8)$ & $139.3(5.8)$ & 0.291 \\
\hline Potassium & $4.5(0.9)$ & $4.5(0.9)$ & $4.6(0.9)$ & 0.355 & $4.6(0.9)$ & $4.5(0.8)$ & 0.586 & $4.7(0.8)$ & $4.4(0.9)$ & 0.002 \\
\hline Chrorine & $103.8(12.0)$ & $103.8(11.5)$ & $103.8(13.2)$ & 0.970 & $104.2(10.2)$ & $102.5(16.6)$ & 0.426 & $102.8(12.7)$ & $104.4(11.6)$ & 0.250 \\
\hline Haemoglobin & $10.7(2.6)$ & $11.0(2.6)$ & $10.0(2.4)$ & 0.0006 & $10.9(2.5)$ & $10.0(2.7)$ & 0.006 & $10.5(2.1)$ & $10.8(2.8)$ & 0.239 \\
\hline VGM & 82.3 (11.9) & $83.7(7.5)$ & $79.5(17.3)$ & 0.058 & $83.0(10.2)$ & $80.4(14.5)$ & 0.223 & $83.5(9.9)$ & 81.5 (13.0) & 0.215 \\
\hline $\mathrm{TCMH}$ & $28.3(7.1)$ & $27.9(3.0)$ & $29.0(11.6)$ & 0.461 & $27.8(2.7)$ & $29.5(12.5)$ & 0.311 & $27.7(2.4)$ & $28.6(8.7)$ & 0.317 \\
\hline Calcemia & $88.5(10.2)$ & $88.6(10.1)$ & $88.3(10.5)$ & 0.865 & $88.6(10.6)$ & $87.9(8.4)$ & 0.553 & $89.0(9.3)$ & 88.1 (10.9) & 0.443 \\
\hline Phosphate & $46.8(21.7)$ & 46.7 (23.8) & $47.0(16.6)$ & 0.913 & $47.8(23.0)$ & $42.1(13.9)$ & 0.043 & $45.0(17.0)$ & $48.1(24.6)$ & 0.291 \\
\hline Uric acid & $86.2(25.8)$ & 86.5 (25.5) & $85.2(26.7)$ & 0.700 & $87.3(26.3)$ & $80.2(22.1)$ & 0.097 & $82.5(23.2)$ & $89.2(27.5)$ & 0.028 \\
\hline Albumin & $33.3(13.7)$ & $33.1(14.5)$ & 33.6 (12.6) & 0.854 & $36.9(14.5)$ & $26.7(9.2)$ & $\begin{array}{l}< \\
0.0001\end{array}$ & $34.9(15.2)$ & $32.4(12.9)$ & 0.345 \\
\hline Total cholesterol & $2.3(1.1)$ & $2.2(1.2)$ & $2.4(1.0)$ & 0.264 & $2.1(0.8)$ & $2.8(1.7)$ & 0.002 & $2.1(0.9)$ & $2.3(1.3)$ & 0.322 \\
\hline HDL cholesterol & $0.54(0.37)$ & $0.53(0.36)$ & $0.56(0.40)$ & 0.642 & $0.54(0.39)$ & $0.53(0.27)$ & 0.767 & $0.52(0.32)$ & $0.55(0.41)$ & 0.116 \\
\hline LDL cholesterol & $1.4(0.7)$ & $1.4(0.7)$ & $1.4(0.6)$ & 0.653 & $1.4(0.6)$ & $1.5(1.1)$ & 0.653 & $1.1(0.6)$ & $1.4(0.7)$ & 0.556 \\
\hline Triglycerides & $1.3(0.9)$ & $1.1(0.7)$ & $1.6(1.1)$ & 0.002 & $1.2(0.7)$ & $1.6(1.4)$ & 0.052 & $1.2(0.7)$ & $1.3(1.0)$ & 0.307 \\
\hline Diabetes, n (\%) & $168(40.0)$ & $118(42.0)$ & $50(36.0)$ & 0.236 & $147(46.1)$ & $21(20.8)$ & $\begin{array}{l}< \\
0.0001\end{array}$ & $168(100)$ & $0(0)$ & $\begin{array}{l}< \\
0.0001\end{array}$ \\
\hline Hypertension, n (\%) & $319(75.9)$ & $221(78.6)$ & $98(70.5)$ & 0.066 & $319(100)$ & $0(0)$ & $\begin{array}{l}< \\
0.0001\end{array}$ & $147(87.5)$ & $172(68.2)$ & $\begin{array}{l}< \\
0.0001\end{array}$ \\
\hline Smoking, n (\%) & $24 / 395(6.1)$ & 21/262 (8.0) & 3/133 (2.3) & 0.025 & 19/297 (6.4) & $5 / 98(5.1)$ & 0.809 & $8 / 155(5.2)$ & $\begin{array}{l}16 / 240 \\
(6.7)\end{array}$ & 0.668 \\
\hline Alcohol, n (\%) & $\begin{array}{l}129 / 388 \\
(33.2)\end{array}$ & $\begin{array}{l}110 / 258 \\
(42.6)\end{array}$ & $\begin{array}{l}19 / 130 \\
(14.6)\end{array}$ & $\begin{array}{l}< \\
0.0001\end{array}$ & $\begin{array}{l}106 / 293 \\
(36.2)\end{array}$ & $\begin{array}{l}23 / 95 \\
(24.2)\end{array}$ & 0.033 & $\begin{array}{l}44 / 152 \\
(28.9)\end{array}$ & $\begin{array}{l}85 / 236 \\
(36.0)\end{array}$ & 0.153 \\
\hline Sedentarity & $\begin{array}{l}163 / 358 \\
(45.5)\end{array}$ & $\begin{array}{l}95 / 240 \\
(39.6)\end{array}$ & $\begin{array}{l}68 / 118 \\
(57.6)\end{array}$ & 0.001 & $\begin{array}{l}134 / 269 \\
(49.8)\end{array}$ & $\begin{array}{l}29 / 89 \\
(32.6)\end{array}$ & 0.005 & $\begin{array}{l}69 / 140 \\
(49.3)\end{array}$ & $\begin{array}{l}94 / 218 \\
(43.1)\end{array}$ & 0.277 \\
\hline HIV infection, n (\%) & $20 / 411(4.9)$ & $7 / 274(2.5)$ & $\begin{array}{l}13 / 137 \\
(9.5)\end{array}$ & 0.009 & $4 / 311(1.3)$ & $\begin{array}{l}16 / 100 \\
(16.0)\end{array}$ & $\begin{array}{l}< \\
0.0001\end{array}$ & $3 / 165(1.8)$ & $\begin{array}{l}17 / 246 \\
(6.9)\end{array}$ & 0.043 \\
\hline Gout, n (\%) & $28 / 411(6.8)$ & 25/275 (9.1) & $3 / 136(2.2)$ & 0.011 & 27/313 (8.6) & $1 / 98(1.0)$ & 0.005 & $\begin{array}{l}10 / 166 \\
(6.0)\end{array}$ & $\begin{array}{l}18 / 245 \\
(7.3)\end{array}$ & 0.692 \\
\hline Stage CKD $(n=403)$ & & & & 0.040 & & & 0.002 & & & 0.013 \\
\hline 1 & $3(0.7)$ & $3(1.1)$ & $0(0)$ & & $1(0.3)$ & $2(2.3)$ & & $0(0)$ & $3(1.3)$ & \\
\hline 2 & $17(4.2)$ & $13(4.8)$ & $4(3.0)$ & & $7(2.2)$ & $10(11.4)$ & & $2(1.2)$ & $15(6.4)$ & \\
\hline 3 & $151(37.5)$ & $111(41.1)$ & $40(30.1)$ & & $116(36.8)$ & $35(39.8)$ & & $70(41.9)$ & $81(34.3)$ & \\
\hline 4 & $124(30.8)$ & $80(29.6)$ & $44(33.1)$ & & $104(33.0)$ & $20(22.7)$ & & $54(32.3)$ & $70(29.7)$ & \\
\hline 5 & 108 (26.8) & $63(23.3)$ & 45 (33.8) & & 87 (27.6) & $21(23.9)$ & & $41(24.5)$ & $67(28.4)$ & \\
\hline
\end{tabular}


Table 1 Baseline characteristics overall and by major subgroups (Continued)

\begin{tabular}{|c|c|c|c|c|c|c|c|c|c|c|}
\hline Characteristics & Overall & Men & Women & $p$ & HTN & No HTN & $P$ & $\mathrm{DM}$ & No DM & $p$ \\
\hline \multicolumn{11}{|l|}{ Treatments } \\
\hline ACE inhibitors & $314(74.8)$ & $123(75.8)$ & $101(72.7)$ & 0.551 & $263(82.4)$ & $51(50.5)$ & $\begin{array}{l}< \\
0.0001\end{array}$ & $137(81.5)$ & $177(70.2)$ & 0.011 \\
\hline Loop diuretics & $130(30.9)$ & 86 (30.6) & 44 (31.6) & 0.823 & $86(27.0)$ & 44 (43.6) & 0.002 & $56(33.3)$ & $74(29.4)$ & 0.391 \\
\hline $\begin{array}{l}\text { Calcium channels } \\
\text { blockers }\end{array}$ & $198(47.1)$ & $125(44.5)$ & $73(52.5)$ & 0.146 & $185(58.0)$ & $13(12.9)$ & $\begin{array}{l}< \\
0.0001\end{array}$ & $81(48.2)$ & $117(46.4)$ & 0.765 \\
\hline Thiazide diuretic & $206(49.0)$ & $142(50.5)$ & $64(46.0)$ & 0.408 & $177(55.5)$ & $29(28.7)$ & $\begin{array}{l}< \\
0.0001\end{array}$ & $91(54.2)$ & $115(45.6)$ & 0.091 \\
\hline Antialdosterone & $17(4.0)$ & $12(4.3)$ & $5(3.6)$ & $>0.999$ & $4(1.2)$ & $13(12.9)$ & $\begin{array}{l}< \\
0.0001\end{array}$ & $4(2.4)$ & $13(5.2)$ & 0.208 \\
\hline ARB & $17(4.0)$ & $10(3.6)$ & $7(5.0)$ & 0.445 & $14(4.3)$ & $3(3.0)$ & 0.773 & $11(6.5)$ & $6(2.4)$ & 0.043 \\
\hline Beta blockers & $54(12.9)$ & $36(12.8)$ & $18(12.9)$ & $>0.999$ & $48(15.0)$ & $6(5.9)$ & 0.017 & $17(10.1)$ & $37(14.7)$ & 0.184 \\
\hline Central agent & $20(4.8)$ & $14(5.0)$ & $6(4.2)$ & $>0.999$ & $20(6.3)$ & $0(0)$ & 0.006 & $9(5.4)$ & $11(4.4)$ & 0.647 \\
\hline Biguanide & $6(1.4)$ & $4(1.4)$ & $2(1.4)$ & $>0.999$ & $6(1.9)$ & $0(0)$ & 0.343 & $6(3.6)$ & $0(0)$ & 0.004 \\
\hline Sulphonamides & 57 (13.6) & $41(14.6)$ & $16(11.5)$ & 0.450 & $50(15.7)$ & $7(6.9)$ & 0.029 & $57(33.9)$ & $0(0)$ & $\begin{array}{l}< \\
0.0001\end{array}$ \\
\hline Glinides & $1(0.2)$ & $1(0.4)$ & $0(0)$ & $>0.999$ & $1(0.3)$ & $0(0)$ & $>0.999$ & $1(0.6)$ & $0(0)$ & 0.400 \\
\hline Insulin & $34(8.1)$ & $22(7.8)$ & $12(8.6)$ & 0.850 & $28(8.8)$ & $6(5.9)$ & 0.411 & $34(20.2)$ & $0(0)$ & $\begin{array}{l}< \\
0.0001\end{array}$ \\
\hline
\end{tabular}

HTN Hypertension, DM Diabetes mellitus, SD Standard deviation, SBP Systolic blood pressure, DPB Diastolic blood pressure, eGFR Estimated Glomerular filtration rate, MCV Mean corpuscular volume, MCH Mean corpuscular haemoglobin, HDL High density lipoprotein, LDL Low density lipoprotein, HIV Human immunedeficiency virus, $A C E$ Angiotensin converting enzyme, ARB Angiotensinogen receptor blockers

Table 2 Trajectory of key variables in the overall sample

\begin{tabular}{|c|c|c|c|c|c|c|}
\hline Variables & Baseline & Month 3 & Month 6 & Month 9 & Month 12 & $p$-value \\
\hline Creatinine & $41.7(3.7)$ & $41.5(3.9)$ & $47.7(4.1)$ & $52.1(4.5)$ & $59.1(5.0)$ & 0.0001 \\
\hline eGFR & $27.0(2.3)$ & $30.3(2.4)$ & $27.7(2.5)$ & $27.0(2.8)$ & $21.5(3.1)$ & 0.003 \\
\hline Urea & $1.00(0.08)$ & $0.97(0.08)$ & $1.04(0.09)$ & $1.01(0.10)$ & $1.21(0.11)$ & 0.178 \\
\hline SBP & $154.4(3.2)$ & $142.1(3.7)$ & $147.3(3.9)$ & $145.5(4.4)$ & $156.0(5.1)$ & $<0.0001$ \\
\hline DBP & $92.4(2.0)$ & $85.4(2.3)$ & $86.3(2.4)$ & $83.9(2.7)$ & $85.6(3.1)$ & $<0.0001$ \\
\hline Sodium & $136.9(1.2)$ & $136.9(1.5)$ & $136.1(1.7)$ & $139.7(2.0)$ & $140.4(2.4)$ & 0.345 \\
\hline Potassium & $4.59(0.12)$ & $4.55(0.13)$ & $4.43(0.14)$ & $4.67(0.16)$ & $4.67(0.20)$ & 0.382 \\
\hline Calcium & $87.4(1.5)$ & $84.6(2.2)$ & $88.1(1.9)$ & $89.0(2.6)$ & $88.0(2.8)$ & 0.558 \\
\hline Haemoglobin & $10.2(0.6)$ & $10.0(0.7)$ & $9.6(0.7)$ & $10.1(0.8)$ & $11.1(0.9)$ & 0.090 \\
\hline $\mathrm{TCMH}$ & $30.2(1.4)$ & $30.4(1.6)$ & $32.2(1.7)$ & $31.4(1.9)$ & $32.0(2.7)$ & 0.535 \\
\hline VGM & $82.1(2.1)$ & $78.8(2.7)$ & $85.9(2.9)$ & 84.9 (3.4) & $91.0(4.5)$ & 0.051 \\
\hline Phosphate & $45.4(3.0)$ & $40.7(5.3)$ & $41.9(4.0)$ & $45.9(5.5)$ & $40.4(5.3)$ & 0.624 \\
\hline Uric acid & 78.3 (3.9) & $67.6(5.4)$ & $70.1(5.1)$ & $68.3(7.2)$ & $65.4(8.2)$ & 0.034 \\
\hline Albumin & $30.5(2.4)$ & $35.3(3.0)$ & $27.8(3.3)$ & $30.5(4.1)$ & $37.0(7.3)$ & 0.125 \\
\hline Total cholesterol & $2.4(0.2)$ & $2.0(0.2)$ & $1.8(0.3)$ & $1.9(0.3)$ & $2.6(0.4)$ & 0.036 \\
\hline HDL cholesterol & $0.51(0.06)$ & $0.36(0.19)$ & $0.30(0.15)$ & $0.44(0.27)$ & $0.53(0.24)$ & 0.685 \\
\hline Triglycerides & $1.7(0.2)$ & $1.3(0.4)$ & $1.6(0.3)$ & $1.5(0.4)$ & $1.4(0.4$ & 0.648 \\
\hline
\end{tabular}

Estimates are mean and standard error of the mean, and are adjusted for age, sex, diabetes, hypertension smoking alcohol consumption, HIV status SBP Systolic blood pressure, DPB Diastolic blood pressure, eGFR Estimated Glomerular filtration rate, $M C H$ Mean corpuscular haemoglobin, $M C V$ Mean corpuscular volume, $H D L$ High density lipoprotein, LDL Low density lipoprotein 


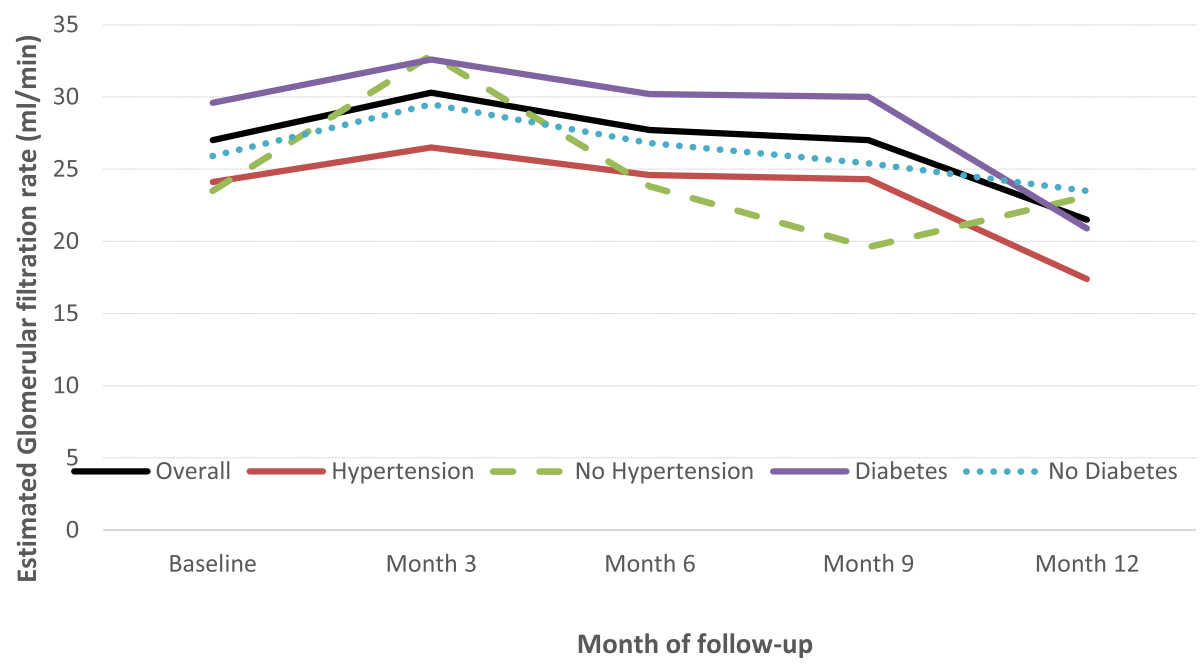

Fig. 1 Trajectory of eGFR overall and by major subgroups. $P$-values for linear trends are 0.003 in the overall sample, 0.058 for participants withand 0.013 for those without diabetes; 0.010 for participants with- and 0.030 for those without hypertension

In its natural history, CKD progresses silently to ESKD and studies have shown that trajectories of glomerular filtration rate (GFR) over time are heterogeneous [3338]. The rate of decline in kidney function is related to the advancement of CKD stages, to risk factors for CKD progression, and treatments $[15,39-41]$. In the present study, more than half of the population was at CKD Stage $4(30.8 \%)$ and Stage 5 (26.8\%) at referral, with men seemed to be referred to the service earlier than women. The main reason of this disparity is that women in this setting would mostly be in financial disadvantage for multiple reasons including non-employment, low income, dependence on the male partner. These are advanced stages of CKD with almost always inexorable progression to ESKD [42-44]. But this progression varies across populations and according to the presence of certain comorbidities [42, 43, 45, 46]. Morgan et al. reported a 1-year cumulative incidence of ESKD of 4.3\% from CKD stage 4 and $49 \%$ from stage 5; and the level of proteinuria was the main predictor of the risk of progressing to ESKD with a median progression time of 9 months for participants with high proteinuria and 19 months for those with lower proteinuria [47]. A potential initial improvement of the kidney function in our sample, likely reflect the effects of treatments adjustment, intensification or initiation by nephrologists at the first visit. In general most patients with CKD in this setting would have been on non-optimal treatments prior to their referral to nephrologists.

Hypertension and diabetes are well-known risks factors for the development and progression of CKD [9, 40, $41,48,49]$. Studies have reported that the rate of eGFR decline was significantly associated with mean blood pressure [50, 51]. Hemmelgarn et al. reported that the decline in eGFR after a 2-years follow-up, was highest among those with diabetes mellitus [52]. Trajectories of kidney function in our sample were mostly similar by diabetes and hypertension status. This likely reflected the challenges of controlling these major CKD risk factors, and not the lack of their effect on CKD progression. This is substantiated for instance by the similarity of the trajectories of systolic and diastolic blood pressure in participants with and without hypertension during follow-up. Hypertension is generally exacerbated in the context of CKD. Therefore, the parallel trajectories of BP levels in participants with and without hypertension in our sample, to some extent reflect the success of therapeutic measures to lower BP in our sub-sample with hypertension. At baseline, a very large proportion of our participants were on BP lowering medications, with $3 / 4$ on Renin angiotensin aldosterone system blockers (RAAS) witch beneficial effects are consistent in the literature [53, 54]. But in the absence of updated data on treatment during follow-up, it is not possible to determine if BP lowering treatments were appropriately intensified during follow-up in our sample.

We did not have data to assess the effects of glycemic control on the trajectory of kidney function in people with diabetes. However, the distribution of baseline glucose control treatment suggests that their intensity was likely not enough to achieve and maintain good glycemic control. Almost all participants with diabetes also had hypertension with differentially high uptake of reno protective drugs such as angiotensin converting enzyme (ACE) inhibitors and angiotensine receptor inhibitors among those with diabetes at baseline (in line 
Table 3 Trajectory of key variables in participants with and without diabetes

\begin{tabular}{|c|c|c|c|c|c|c|c|c|}
\hline Variables & Subgroup & Baseline & Month 3 & Month 6 & Month 9 & Month 12 & $p$-value & Interaction $p$ \\
\hline \multirow[t]{2}{*}{ Creatinine } & Diabetes & $35.6(8.3)$ & $35.1(8.4)$ & $40.9(8.7)$ & $47.5(9.2)$ & $60.8(9.7)$ & 0.001 & 0.334 \\
\hline & No diabetes & $42.8(4.7)$ & $43.1(5.0)$ & $49.7(5.3)$ & $52.8(5.7)$ & $53.5(6.7)$ & 0.058 & \\
\hline \multirow[t]{2}{*}{ eGFR } & Diabetes & $29.6(4.2)$ & $32.6(4.3)$ & $30.2(4.5)$ & $30.0(4.9)$ & $20.9(5.1)$ & 0.013 & 0.646 \\
\hline & No diabetes & $25.9(2.8)$ & $29.5(3.1)$ & $26.8(3.2)$ & $25.4(3.6)$ & $23.5(4.2)$ & 0.205 & \\
\hline \multirow[t]{2}{*}{ Urea } & Diabetes & $1.13(0.17)$ & $1.07(0.18)$ & $1.09(0.19)$ & $1.11(0.22)$ & $1.34(0.21)$ & 0.531 & 0.862 \\
\hline & No diabetes & $1.02(0.10)$ & $1.02(0.10)$ & $1.10(0.11)$ & $1.04(0.12)$ & $1.20(0.15)$ & 0.452 & \\
\hline \multirow[t]{2}{*}{ SBP } & Diabetes & $153.1(8.4)$ & $145.6(8.7)$ & $150.8(9.1)$ & $162.6(9.9)$ & $149.4(10.3)$ & 0.030 & 0.006 \\
\hline & No diabetes & 153.5 (3.6) & $139.2(8.3)$ & $144.6(4.6)$ & $136.3(5.0)$ & $143.9(6.4)$ & $<0.0001$ & \\
\hline \multirow[t]{2}{*}{ DBP } & Diabetes & $86.2(8.4)$ & $81.2(4.6)$ & $83.8(4.9)$ & $83.6(5.5)$ & $81.7(5.6)$ & 0.193 & 0.174 \\
\hline & No diabetes & $96.9(2.4)$ & $88.9(2.7)$ & $88.9(2.9)$ & $85.2(3.2)$ & $88.7(4.0)$ & $<0.0001$ & \\
\hline \multirow[t]{2}{*}{ Sodium } & Diabetes & 127.6 (3.9) & $130.2(4.1)$ & $127.1(4.4)$ & $129.6(5.1)$ & $131.9(5.2)$ & 0.430 & 0.298 \\
\hline & No diabetes & $138.8(1.3)$ & $136.4(1.9)$ & $137.7(1.9)$ & $141.6(2.2)$ & 141.7 (3.1) & 0.256 & \\
\hline \multirow[t]{2}{*}{ Potassium } & Diabetes & $4.8(0.3)$ & $4.8(0.3)$ & $4.6(0.3)$ & $4.9(0.3)$ & $5.0(0.4)$ & 0.542 & 0.935 \\
\hline & No diabetes & $4.4(0.1)$ & $4.3(0.2)$ & $4.2(0.2)$ & $4.4(0.2)$ & $4.4(0.3)$ & 0.552 & \\
\hline \multirow[t]{2}{*}{ Calcium } & Diabetes & $84.2(4.0)$ & $74.7(4.8)$ & $84.9(4.7)$ & $84.4(5.7)$ & $84.2(5.4)$ & 0.098 & 0.216 \\
\hline & No diabetes & $88.9(1.7)$ & $89.1(2.6)$ & $88.9(2.2)$ & $91.9(2.9)$ & $90.4(3.6)$ & 0.848 & \\
\hline \multirow[t]{2}{*}{ Haemoglobin } & Diabetes & $10.0(0.7)$ & $9.0(0.9)$ & $9.1(1.1)$ & $10.3(1.5)$ & $12(1.2)$ & 0.022 & 0.005 \\
\hline & No diabetes & $11.2(0.6)$ & $10.7(0.7)$ & $10.3(0.7)$ & $10.0(0.7)$ & $9.8(0.9)$ & 0.079 & \\
\hline \multirow[t]{2}{*}{ TCMH } & Diabetes & $27.2(1.7)$ & $27.4(1.8)$ & $28.5(1.8)$ & $28.2(2.0)$ & $28.7(2.2)$ & 0.582 & 0.981 \\
\hline & No diabetes & $30.8(1.8)$ & $29.7(2.5)$ & $32.5(2.6)$ & $31.5(3.1)$ & $32.2(5.9)$ & 0.908 & \\
\hline \multirow[t]{2}{*}{ VGM } & Diabetes & $84.2(3.0)$ & $85.3(4.0)$ & $93.1(4.3)$ & $90.3(5.1)$ & $91.8(4.7)$ & 0.069 & 0.325 \\
\hline & No diabetes & $81.2(2.6)$ & $75.6(3.5)$ & 80.7 (3.9) & $82.0(4.4)$ & $92.3(8.3)$ & 0.260 & \\
\hline \multirow[t]{2}{*}{ Phosphate } & Diabetes & $47.3(6.2)$ & $52.1(8.3)$ & $43.2(8.1)$ & $50.7(9.1)$ & $48.3(7.8)$ & 0.707 & 0.586 \\
\hline & No diabetes & $46.4(4.4)$ & $39.6(7.2)$ & $44.7(5.7)$ & $51.3(7.5)$ & $35.0(9.0)$ & 0.433 & \\
\hline \multirow[t]{2}{*}{ Uric acid } & Diabetes & $73.3(6.5)$ & $66.1(7.3)$ & $69.1(8.6)$ & $72.3(11.3)$ & $78.0(14.1)$ & 0.431 & 0.407 \\
\hline & No diabetes & $80.9(4.5)$ & $68.5(7.2)$ & $69.0(6.2)$ & $63.0(8.9)$ & $56.4(9.8)$ & 0.021 & \\
\hline \multirow[t]{2}{*}{ Albumin } & Diabetes & $26.4(2.9)$ & $30.6(4.5)$ & $19.0(6.9)$ & $24.2(13.3)$ & 21.6 (13.3) & 0.715 & 0.480 \\
\hline & No diabetes & $33.0(3.3)$ & $38.1(3.9)$ & $31.2(4.2)$ & $33.7(4.9)$ & $48.9(10.1)$ & 0.150 & \\
\hline \multirow[t]{2}{*}{ Total cholesterol } & Diabetes & $1.8(0.3)$ & $0.8(0.4)$ & $1.3(0.4)$ & - & $2.3(0.4)$ & 0.307 & 0.889 \\
\hline & No diabetes & $2.6(0.2)$ & $2.3(0.3)$ & $1.9(0.3)$ & $2.1(0.3)$ & $2.6(0.6)$ & 0.182 & \\
\hline \multirow[t]{2}{*}{ HDL cholesterol } & Diabetes & $0.34(0.15)$ & $0.15(0.21)$ & $0.21(0.21)$ & - & $0.28(0.26)$ & 0.626 & 0.852 \\
\hline & No diabetes & $0.61(0.08)$ & $0.34(0.46)$ & $0.31(0.22)$ & $0.57(0.33)$ & $0.92(0.48)$ & 0.733 & \\
\hline \multirow[t]{2}{*}{ Triglycerides } & Diabetes & $0.93(0.21)$ & $0.84(0.30)$ & $0.98(0.32)$ & - & $0.70(0.40)$ & 0.846 & 0.119 \\
\hline & No diabetes & $2.01(0.22)$ & $-0.46(0.48)$ & $1.63(0.50)$ & $1.63(0.41)$ & $1.58(0.57)$ & 0.118 & \\
\hline
\end{tabular}

Estimates are mean and standard error of the mean, and are adjusted for age, sex, diabetes, hypertension smoking alcohol consumption, HIV status SBP Systolic blood pressure, DPB Diastolic blood pressure, eGFR Estimated Glomerular filtration rate, $M C H$ Mean corpuscular haemoglobin, $M C V$ Mean corpuscular volume, $H D L$ High density lipoprotein, LDL Low density lipoprotein

with guidelines) [53, 55-57]. In general however, BP levels and SBP in particular are the stronger predictor of renal outcome in people with diabetes [57, 58]. Because of the late referral, biological perturbations related to CKD were already apparent in our sample at baseline including hematological abnormalities and low serum albumin. These abnormalities mostly persisted during follow-up although some late improvements were observed particularly for total hemoglobin levels in participants with diabetes, and serum albumin in the overall sample. In the absence of data on specific treatments targeting those attributes, we can speculate that the observed late improvement was likely due to the selection process. It is indeed an expectation that during follow-up participants with severe disease at baseline (advanced stage CKD) would fall out (through 
Table 4 Trajectory of key variables in participants with and without hypertension

\begin{tabular}{|c|c|c|c|c|c|c|c|c|}
\hline Variables & Subgroup & Baseline & Month 3 & Month 6 & Month 9 & Month 12 & $p$-value & Interaction $p$ \\
\hline \multirow[t]{2}{*}{ Creatinine } & Hypertension & $41.5(6.9)$ & $41.9(7.1)$ & $46.7(7.2)$ & $50.9(7.4)$ & $58.1(7.8$ & 0.0028 & 0.613 \\
\hline & No hypertension & $44.7(6.1)$ & $41.7(6.8)$ & $55.0(7.4)$ & $59.7(8.3)$ & $64.3(10.2)$ & 0.042 & \\
\hline \multirow[t]{2}{*}{ eGFR } & Hypertension & 24.1 (3.3) & $26.5(3.4)$ & $24.6(3.5)$ & $24.3(3.7)$ & $17.4(3.9)$ & 0.010 & 0.054 \\
\hline & No hypertension & 23.5 (3.6) & $32.9(4.3)$ & $23.8(5.0)$ & $19.6(6.0)$ & $23.1(6.9)$ & 0.030 & \\
\hline \multirow[t]{2}{*}{ Urea } & Hypertension & $1.01(0.12)$ & $1.06(0.12)$ & $1.04(0.13)$ & $1.00(0.14)$ & $1.22(0.14)$ & 0.253 & 0.273 \\
\hline & No hypertension & $1.09(0.13)$ & $0.95(0.14)$ & $1.20(0.16)$ & $1.16(0.24)$ & $1.15(0.24)$ & 0.188 & \\
\hline \multirow[t]{2}{*}{ SBP } & Hypertension & $163.9(5.3)$ & $151.0(5.7)$ & $156.1(5.8)$ & $159.8(6.4)$ & $153.5(6.9)$ & 0.0003 & 0.355 \\
\hline & No hypertension & $138.9(6.4)$ & $130.9(7.1)$ & $135.0(7.8)$ & $125.1(8.4)$ & $138.1(10.3)$ & 0.099 & \\
\hline \multirow[t]{2}{*}{ DBP } & Hypertension & 94.7 (3.2) & 87.5 (3.5) & 89.8 (3.6) & $87.5(3.9)$ & $87.5(4.1)$ & 0.0002 & 0.713 \\
\hline & No hypertension & 88.7 (3.6) & $82.6(4.0)$ & $82.0(4.6)$ & $80.2(4.8)$ & $85.4(5.9)$ & 0.043 & \\
\hline \multirow[t]{2}{*}{ Sodium } & Hypertension & $138.0(2.4)$ & $135.1(2.7)$ & $138.3(2.9)$ & $140.6(3.2)$ & $141.6(3.7)$ & 0.207 & 0.388 \\
\hline & No hypertension & $138.4(1.9)$ & $139.8(2.2)$ & $135.3(2.5)$ & $141.5(3.1)$ & $141.9(3.8)$ & 0.221 & \\
\hline \multirow[t]{2}{*}{ Potassium } & Hypertension & $4.54(0.20)$ & $4.54(0.21)$ & $4.31(0.22)$ & $4.53(0.24)$ & $4.59(0.27)$ & 0.241 & 0.011 \\
\hline & No hypertension & $5.01(0.24)$ & $4.60(0.30)$ & $4.98(0.33)$ & $5.67(0.41)$ & $5.08(0.52)$ & 0.154 & \\
\hline \multirow[t]{2}{*}{ Calcium } & Hypertension & 88.7 (2.6) & $83.2(3.2)$ & 89.8 (3.1) & $89.4(3.5)$ & $90.0(3.7)$ & 0.171 & 0.402 \\
\hline & No hypertension & $87.8(2.5)$ & $91.6(3.6)$ & $87.2(3.4)$ & $94.5(5.3)$ & $81.8(6.1)$ & 0.339 & \\
\hline \multirow[t]{2}{*}{ Haemoglobin } & Hypertension & $10.8(0.8)$ & $10.2(0.8)$ & $9.9(0.8)$ & $9.7(0.9)$ & $10.5(0.9)$ & 0.100 & 0.058 \\
\hline & No hypertension & $9.9(0.8)$ & $9.5(1.0)$ & $9.1(2.1)$ & $10.7(2.5)$ & $10.1(1.9)$ & 0.960 & \\
\hline \multirow[t]{2}{*}{$\mathrm{TCMH}$} & Hypertension & $29.6(1.6)$ & $29.7(1.9)$ & $33.2(1.9)$ & $30.6(2.3)$ & $31.6(3.0)$ & 0.153 & 0.351 \\
\hline & No hypertension & $28.8(3.9)$ & $29.1(4.3)$ & $23.4(4.7)$ & $26.3(5.1)$ & $28.4(6.7)$ & 0.424 & \\
\hline \multirow[t]{2}{*}{ VGM } & Hypertension & 84.3 (3.3) & $80.2(3.8)$ & $88.0(4.0)$ & $85.3(4.7)$ & $92.2(6.1)$ & 0.165 & 0.889 \\
\hline & No hypertension & $77.8(4.0)$ & $75.2(5.4)$ & $81.6(5.9)$ & $83.2(6.4)$ & $92.2(8.3)$ & 0.425 & \\
\hline \multirow[t]{2}{*}{ Phosphate } & Hypertension & $52.6(5.0)$ & $51.7(6.5)$ & 49.7 (5.8) & $54.4(6.9)$ & $51.2(7.0)$ & 0.902 & 0.860 \\
\hline & No hypertension & $44.3(4.7)$ & $37.4(14.9)$ & $40.5(7.1)$ & $67.6(15.1)$ & 34.7 (10.4) & 0.516 & \\
\hline \multirow[t]{2}{*}{ Uric acid } & Hypertension & $92.4(6.2)$ & $82.2(7.3)$ & $84.3(7.1)$ & $83.6(8.7)$ & 79.6 (9.4) & 0.089 & 0.872 \\
\hline & No hypertension & $72.5(5.9)$ & $59.0(8.6)$ & $71.9(11.3)$ & $65.2(20.4)$ & - & 0.347 & \\
\hline \multirow[t]{2}{*}{ Albumin } & Hypertension & $33.8(4.0)$ & $30.6(6.6)$ & $30.9(5.8)$ & $28.2(7.6)$ & $27.1(13.1)$ & 0.825 & 0.246 \\
\hline & No hypertension & $25.9(2.8)$ & $32.0(3.4)$ & $23.4(4.3)$ & $28.3(5.1)$ & $41.3(9.9)$ & 0.078 & \\
\hline \multirow[t]{2}{*}{ Total cholesterol } & Hypertension & $2.41(0.20)$ & $2.00(0.25)$ & $1.87(0.29)$ & $1.96(0.34)$ & $2.66(0.42)$ & 0.114 & 0.916 \\
\hline & No hypertension & $2.24(0.33)$ & $2.81(0.78)$ & $0.15(1.19)$ & $1.72(0.96)$ & $1.89(0.86)$ & 0.513 & \\
\hline \multirow[t]{2}{*}{ HDL cholesterol } & Hypertension & $0.48(0.12)$ & $0.32(0.17)$ & $0.37(0.17)$ & $0.41(0.33)$ & $0.50(0.22)$ & 0.633 & 0.761 \\
\hline & No hypertension & $0.57(0.13)$ & - & - & $0.00009(0.35)$ & - & 0.158 & \\
\hline \multirow[t]{2}{*}{ Triglycerides } & Hypertension & $1.83(0.13)$ & $0.92(0.34)$ & $1.53(0.31)$ & $1.82(0.39)$ & $1.51(0.34)$ & 0.262 & 0.956 \\
\hline & No hypertension & $1.32(0.47)$ & - & - & $1.83(1.79)$ & - & Not computable & \\
\hline
\end{tabular}

Estimates are mean and standard error of the mean, and are adjusted for age, sex, diabetes, hypertension smoking alcohol consumption, HIV status SBP Systolic blood pressure, DPB Diastolic blood pressure, eGFR Estimated Glomerular filtration rate, $M C H$ Mean corpuscular haemoglobin, $M C V$ Mean corpuscular volume, $H D L$ High density lipoprotein, LDL Low density lipoprotein, NC Not computable

initiation of renal replacement therapy for instance), and therefore mostly healthier patients and better biological profile would remain in the cohort with extended follow-up.

\section{Strengths and limitations}

Our study including a representative sample size $(n=$ 420) of patients with CKD, has provided for the first time, evidence on the trajectory of kidney function and some major risk factor over time in newly referred patients in SSA using linear regression model. An assessment of the rate of decline of renal function is important but complex because renal decline is rarely a linear phenomenon and there is no gold standard methodology. A major limitation to this study is the retrospective nature of data collection with missing follow up 
data for some risk markers that could have influenced our results. Also proteinuria a major progression factor for CKD was not included as follow up data in this study. We could not evaluate the association between kidney function over time with onset of ESKD and mortality. Also patient on dialysis were excluded. We did not have data on treatments uptake and changes during follow-up, and therefore, could not fully account for their possible effect on the trajectories of investigated markers. Lastly, the current study was not based on power calculation. However, we used the totality of eligible observations at our center during the study period.

\section{Conclusion}

In conclusion, our study provides additional evidence that patients with CKD in African settings are referred late to nephrologists, with an advanced stage of CKD. This likely translates into a less beneficial effect of specialised care on the course of the disease, with kidney function inexorably declining further within the first year of follow-up, regardless of underlying comorbidities and risk factors. To what extent this progression reflects non-optimal uptake of CKD modifying therapies at baseline, and their intensification during follow-up, need to be investigated in future studies. Such information is needed to optimise the prevention of CKD progression in this setting.

\section{Abbreviations}

CKD: Chronic Kidney Disease; eGFR: Estimated Glomerular Filtration Rate; ESKD: End Stage Kidney Disease; RRT: Renal Replacement Therapy; CVD: Cardiovascular Disease; SSA: Sub-Saharan Africa; DGH: Douala General Hospital; MDRD: Modification of Diet in Renal Disease; KDIGO: Kidney Disease Improving Global Outcome; SBP: Systolic Blood Pressure; DBP: Diastolic Blood Pressure

\section{Acknowledgments}

None.

\begin{abstract}
Authors' contributions
$H M P, C S P$, and KAP: Study conception and design. HMP led the study, drafted the manuscript with contribution of KAP, CSP, AEG, DMS. KAP: data analysis. $D H$ : data collection with supervision of KFF, DMS. All authors contributed to data interpretation and revision of the manuscript. HMP, KAP and CSP are the guarantors of this study. The authors read and approved the final manuscript
\end{abstract}

\section{Funding}

None.

\section{Availability of data and materials}

Data are available from the corresponding Author.

\section{Ethics approval and consent to participate}

Ethical approval was obtained from the ethical committee board of the Douala University and administrative authorization from the DGH. Consent to participate wasn't required since this study was based on available health records, with no further contact with participants.

\section{Consent for publication}

Not required.

\section{Competing interests}

The authors declare that they have no competing interests.

\section{Author details}

'Department of internal medicine Douala General Hospital Cameroon Faculty of medicine and pharmaceutical science, University of Douala, Douala, Cameroon. ${ }^{2}$ Non-Communicable Diseases Research Unit, South African Medical Research Council, Cape Town, South Africa. ${ }^{3}$ Faculty of medicine and biomedical sciences, University of Yaoundé I, Yaounde, Cameroon. ${ }^{4}$ Higher Institute of Health Sciences, Université des Montagnes, Bangangte, Cameroon. ${ }^{5}$ Department of internal medicine Douala General Hospital Cameroon, Faculty of medicine and pharmaceutical science, University of Douala, Douala, Cameroon. 'Department of internal medicine Yaounde general hospital Cameroon, Faculty of medicine and biomedical sciences, University of Yaoundé I, Yaounde, Cameroon. ${ }^{7}$ Department of Internal Medicine Douala General Hospital Cameroon, Faculty of Medicine and Pharmaceutical Sciences, University of Dschang, Dschang, Cameroon.

Received: 22 January 2019 Accepted: 9 March 2020

Published online: 18 March 2020

\section{References}

1. Hill NR, Fatoba ST, Oke JL, Hirst JA, O'Callaghan CA, Lasserson DS, et al. Global prevalence of chronic kidney Disease - a systematic review and meta-analysis. PLoS One. 2016;11(7):e0158765.

2. Lozano R, Naghavi M, Foreman K, Lim S, Shibuya K, Aboyans V, et al. Global and regional mortality from 235 causes of death for 20 age groups in 1990 and 2010: a systematic analysis for the global burden of Disease study 2010. Lancet. 2012 Dec 15;380(9859):2095-128.

3. Levey AS, Coresh J, Balk E, Kausz AT, Levin A, Steffes MW, et al. National Kidney Foundation practice guidelines for chronic kidney disease: evaluation, classification, and stratification. Ann Intern Med. 2003;139(2):137-47.

4. Stevens PE, Levin A. Kidney Disease: improving global outcomes chronic kidney Disease guideline development work group members. Evaluation and management of chronic kidney disease: synopsis of the kidney disease: improving global outcomes 2012 clinical practice guideline. Ann Intern Med. 2013;158(11):825-30.

5. Fried LF, Shlipak MG, Crump C, Bleyer AJ, Gottdiener JS, Kronmal RA, et al. Renal insufficiency as a predictor of cardiovascular outcomes and mortality in elderly individuals. J Am CollCardiol. 2003;41(8):1364-72.

6. Peralta CA, Katz R, DeBoer I, Ix J, Sarnak M, Kramer H, et al. Racial and ethnic differences in kidney function decline among persons without chronic kidney disease. J Am SocNephrol. 2011;22(7):1327-34.

7. Collins AJ, Foley RN, Chavers B, Gilbertson D, Herzog C, Johansen K, et al. United States Renal Data System 2011 Annual Data Report: Atlas of chronic kidney disease \& end-stage renal disease in the United States. Am J Kidney Dis. 2012;59(1 Suppl 1):A7, e1-420.

8. Peralta CA, Risch N, Lin F, Shlipak MG, Reiner A, Ziv E, et al. The Association of African Ancestry and elevated creatinine in the coronary artery risk development in Young adults (CARDIA) study. Am J Nephrol. 2010;31(3): 202-8.

9. Young BA, Maynard C, Boyko EJ. Racial differences in diabetic nephropathy, cardiovascular disease, and mortality in a national population of veterans. Diabetes Care. 2003;26(8):2392-9.

10. Foster MC, Coresh J, Fornage M, Astor BC, Grams M, Franceschini N, et al. APOL1 variants associate with increased risk of CKD among African Americans. J Am SocNephrol. 2013;24(9):1484-91.

11. Genovese G, Friedman DJ, Ross MD, Lecordier L, Uzureau P, Freedman BI, et al. Association of trypanolytic ApoL1 variants with kidney disease in African Americans. Science. 2010;329(5993):841-5.

12. Kaze AD, Schutte AE, Erqou S, Kengne AP, Echouffo-Tcheugui JB. Prevalence of hypertension in older people in Africa: a systematic review and metaanalysis. J Hypertens. 2017;35(7):1345-52.

13. Codreanu I, Perico N, Sharma SK, Schieppati A, Remuzzi G. Prevention programmes of progressive renal disease in developing nations. Nephrology (Carlton). 2006;11(4):321-8.

14. Kiberd BA, Clase CM. Cumulative risk for developing end-stage renal disease in the US population. J Am SocNephrol. 2002;13(6):1635-44.

15. Alves TP, Wang X, Wright JT, Appel LJ, Greene T, Norris K, et al. Rate of ESRD exceeds mortality among African Americans with hypertensive nephrosclerosis. J Am SocNephrol. 2010;21(8):1361-9. 
16. Peralta CA, Vittinghoff E, Bansal N, Jacobs D, Muntner P, Kestenbaum B, et al. Trajectories of kidney function decline in young black and white adults with preserved GFR: results from the coronary artery risk development in Young adults (CARDIA) study. Am J Kidney Dis. 2013;62(2): 261-6.

17. Ene-lordache B, Perico N, Bikbov B, Carminati S, Remuzzi A, Perna A, et al. Chronic kidney disease and cardiovascular risk in six regions of the world (ISN-KDDC): a cross-sectional study. Lancet Glob Health. 2016;4(5):e307-19.

18. Stanifer JW, Jing B, Tolan S, Helmke N, Mukerjee R, Naicker S, et al. The epidemiology of chronic kidney disease in sub-Saharan Africa: a systematic review and meta-analysis. Lancet Glob Health. 2014;2(3):e174-81.

19. Sumaili EK, Krzesinski J-M, Cohen EP, Nseka NM. Epidemiology of chronic kidney disease in the Democratic Republic of Congo: review of crosssectional studies from Kinshasa, the capital. NephrolTher. 2010;6(4):232-9.

20. Werfalli M, Musekiwa A, Engel ME, Ross I, Kengne AP, Levitt NS. The prevalence of type 2 diabetes mellitus among older people in Africa: a systematic review study protocol. BMJ Open. 2014:4(6):e004747.

21. Naicker S. End-stage renal disease in sub-Saharan Africa. Ethn Dis. 2009;19(1 Suppl 1):S1-13-5.

22. Halle MP, Luma H, Temfack E, Vanessa T, Kaze F, Ashuntantang G, et al. Prevalence of hepatitis B surface antigen and anti-HIV antibodies among patients on maintenance haemodialysis in Douala, Cameroon. Health Sci Dis. 2013 Sep;1:14.

23. Halle MP, Takongue C, Kengne AP, Kaze FF, Ngu KB. Epidemiological profile of patients with end stage renal disease in a referral hospital in Cameroon. BMC Nephrology [Internet]. 2015;16(1) Available from: http://www. biomedcentral.com/1471-2369/16/59, [cited 2015 Dec 24].

24. Halle MP, Kengne AP, Ashuntantang G. Referral of patients with kidney impairment for specialist care in a developing country of sub-Saharan Africa. Ren Fail. 2009:31(5):341-8.

25. Halle MP, Tsinga L, Fottsoh AF, Kaze FF, Sone AM, Ashuntantang G. Does Timing of Nephrology Referral Influence Outcome among Patients on Maintenance Hemodialysis in Cameroon? Health Sci Dis [Internet]. 2017; 18(3) Available from: http://www.hsd-fmsb.org/index.php/hsd/article/view/ 862.

26. Liyanage $T$, Ninomiya $T$, Jha V, Neal B, Patrice HM, Okpechi I, et al. Worldwide access to treatment for end-stage kidney disease: a systematic review. Lancet [Internet]. 2015:385(9981):1975-82 Available from: http:// linkinghub.elsevier.com/retrieve/pii/S0140673614616019, [cited 2016 Apr 26].

27. Halle MP, Jimkap NN, Kaze FF, Fouda H, Belley EP. Ashuntantang G. Cost of care for patients on maintenance haemodialysis in public facilities in Cameroon. Afr J Nephrol [Internet]. 2017;20(1):230-7 Available from: http:// www.journals.ac.za/index.php/ajn/article/view/2548, [cited 2018 Jan 24].

28. Ashuntantang G, Osafo C, Olowu WA, Arogundade F, Niang A, Porter J, et al. Outcomes in adults and children with end-stage kidney disease requiring dialysis in sub-Saharan Africa: a systematic review. Lancet Global Health [Internet]. 2017;5(4):e408-17 Available from: http://linkinghub.elsevier. com/retrieve/pii/S2214109X17300578, [cited 2017 Oct 31].

29. Halle MP, Ashuntantang G, Kaze FF, Takongue C, Kengne A-P. Fatal outcomes among patients on maintenance haemodialysis in sub-Saharan Africa: a 10-year audit from the Douala general Hospital in Cameroon. BMC Nephrol. 2016;17(1):165.

30. Levey AS, Coresh J, Greene T, Stevens LA, Zhang YL, Hendriksen S, et al. Using standardized serum creatinine values in the modification of diet in renal disease study equation for estimating glomerular filtration rate. Ann Intern Med. 2006;145(4):247-54.

31. Schwartz GJ, Brion LP, Spitzer A. The use of plasma creatinine concentration for estimating glomerular filtration rate in infants, children, and adolescents. PediatrClin North Am. 1987;34(3):571-90.

32. Disease K. Improving Global Outcomes (KDIGO) CKD Work Group. KDIGO 2012 Clinical Practice Guideline for the Evaluation and Management of Chronic Kidney Disease. Kidney Int Suppl. 2013;3:1-150.

33. Lemley KV, Boothroyd DB, Blouch KL, Nelson RG, Jones LI, Olshen RA, et al. Modeling GFR trajectories in diabetic nephropathy. Am J Physiol Renal Physiol. 2005 Oct;289(4):F863-70.

34. O'Hare AM, Batten A, Burrows NR, Pavkov ME, Taylor L, Gupta I, et al. Trajectories of kidney function decline in the 2 years before initiation of long-term dialysis. Am J Kidney Dis. 2012;59(4):513-22.

35. Li L, Astor BC, Lewis J, Hu B, Appel LJ, Lipkowitz MS, et al. Longitudinal progression trajectory of GFR among patients with CKD. Am J Kidney Dis. 2012;59(4):504-12.
36. Leffondre K, Boucquemont J, Tripepi G, Stel VS, Heinze G, Dunkler D. Analysis of risk factors associated with renal function trajectory over time: a comparison of different statistical approaches. Nephrol Dial Transplant. 2015; 30(8):1237-43.

37. Xie Y, Bowe B, Xian H, Balasubramanian S, Al-Aly Z. Estimated GFR trajectories of people entering CKD stage 4 and subsequent kidney Disease outcomes and mortality. Am J Kidney Dis. 2016;68(2):219-28.

38. Collister D, Ferguson T, Komenda P, Tangri N. The patterns, risk factors, and prediction of progression in chronic kidney Disease: a narrative review. SeminNephrol. 2016;36(4):273-82

39. Levin A, Djurdjev O, Beaulieu M, Er L. Variability and risk factors for kidney disease progression and death following attainment of stage 4 CKD in a referred cohort. Am J Kidney Dis. 2008;52(4):661-71.

40. Appel LJ, Wright JT, Greene T, Agodoa LY, Astor BC, Bakris GL, et al. Intensive blood-pressure control in hypertensive chronic kidney disease. $\mathrm{N}$ Engl J Med. 2010;363(10):918-29.

41. Young BA, Katz R, Boulware LE, Kestenbaum B, de Boer $\mid H$, Wang W, et al. Risk factors for rapid kidney function decline among African Americans: the Jackson heart study (JHS). Am J Kidney Dis. 2016;68(2):229-39.

42. Johnson ES, Thorp ML, Platt RW, Smith DH. Predicting the risk of dialysis and transplant among patients with CKD: a retrospective cohort study. Am J Kidney Dis. 2008;52(4):653-60.

43. Keith DS, Nichols GA, Gullion CM, Brown JB, Smith DH. Longitudinal followup and outcomes among a population with chronic kidney disease in a large managed care organization. Arch Intern Med. 2004;164(6):659-63.

44. Ishani A, Grandits GA, Grimm RH, Svendsen KH, Collins AJ, Prineas RJ, et al. Association of single measurements of dipstick proteinuria, estimated glomerular filtration rate, and hematocrit with 25-year incidence of endstage renal disease in the multiple risk factor intervention trial. J Am SocNephrol. 2006:17(5):1444-52.

45. Xue JL, Eggers PW, Agodoa LY, Foley RN, Collins AJ. Longitudinal study of racial and ethnic differences in developing end-stage renal disease among aged medicare beneficiaries. J Am SocNephrol. 2007;18(4):1299-306.

46. Iseki K, Iseki C, Ikemiya Y, Fukiyama K. Risk of developing end-stage renal disease in a cohort of mass screening. Kidney Int. 1996;49(3):800-5.

47. Grams ME, Li L, Greene TH, Tin A, Sang Y, Kao WHL, et al. Estimating time to ESRD using kidney failure risk equations: results from the African American study of kidney Disease and hypertension (AASK). Am J Kidney Dis. 2015; 65(3):394-402.

48. de Boer $\mathrm{H}$, Rue TC, Hall YN, Heagerty PJ, Weiss NS, Himmelfarb J. Temporal trends in the prevalence of diabetic kidney disease in the United States. JAMA. 2011;305(24):2532-9.

49. Hanratty R, Chonchol M, Miriam Dickinson L, Beaty BL, Estacio RO, MacKenzie TD, et al. Incident chronic kidney disease and the rate of kidney function decline in individuals with hypertension. Nephrol Dial Transplant [Internet]. 2010;25(3):801-7 Available from: https://academic.oup.com/ndt/ article-lookup/doi/10.1093/ndt/gfp534, [cited 2018 Dec 6].

50. Lindeman RD, Tobin JD, Shock NW. Association between blood pressure and the rate of decline in renal function with age. Kidney Int. 1984;26(6): $861-8$.

51. Imai E, Horio M, Yamagata K, Iseki K, Hara S, Ura N, et al. Slower decline of glomerular filtration rate in the Japanese general population: a longitudinal 10-year follow-up study. Hypertens Res. 2008;31(3):433-41.

52. Hemmelgarn BR, Zhang J, Manns BJ, Tonelli M, Larsen E, Ghali WA, et al Progression of kidney dysfunction in the community dwelling elderly. Kidney Int. 2006;69(12):2155-61.

53. Lewis EJ, Hunsicker LG, Clarke WR, Berl T, Pohl MA, Lewis JB, et al. Renoprotective effect of the angiotensin-receptor antagonist irbesartan in patients with nephropathy due to type 2 diabetes. N Engl J Med. 2001; 345(12):851-60

54. Zannad F, Kessler M, Lehert P, Grünfeld JP, Thuilliez C, Leizorovicz A, et al. Prevention of cardiovascular events in end-stage renal disease: results of a randomized trial of fosinopril and implications for future studies. Kidney Int. 2006:70(7):1318-24.

55. Keane WF, Brenner BM, de Zeeuw D, Grunfeld J-P, McGill J, Mitch WE, et al. The risk of developing end-stage renal disease in patients with type 2 diabetes and nephropathy: the RENAAL study. Kidney Int. 2003;63(4):1499507.

56. Brenner BM, Cooper ME, de Zeeuw D, Keane WF, Mitch WE, Parving HH, et al. Effects of losartan on renal and cardiovascular outcomes in patients with type 2 diabetes and nephropathy. N Engl J Med. 2001;345(12):861-9. 
57. Bakris GL, Weir MR, Shanifar S, Zhang Z, Douglas J, van Dijk DJ, et al. Effects of blood pressure level on progression of diabetic nephropathy: results from the RENAAL study. Arch Intern Med. 2003;163(13):1555-65.

58. Hirayama A, Konta T, Kamei K, Suzuki K, Ichikawa K, Fujimoto S, et al. Blood pressure, proteinuria, and renal function decline: associations in a large community-based population. Am J Hypertens. 2015;28(9):1150-6.

\section{Publisher's Note}

Springer Nature remains neutral with regard to jurisdictional claims in published maps and institutional affiliations.

Ready to submit your research? Choose BMC and benefit from:

- fast, convenient online submission

- thorough peer review by experienced researchers in your field

- rapid publication on acceptance

- support for research data, including large and complex data types

- gold Open Access which fosters wider collaboration and increased citations

- maximum visibility for your research: over $100 \mathrm{M}$ website views per year

At $\mathrm{BMC}$, research is always in progress.

Learn more biomedcentral.com/submissions 